\title{
Abrasion model of downstream changes in grain shape and size along the Williams River, Australia
}

\author{
Tímea Szabó, ${ }^{1}$ Stephen Fityus, ${ }^{2}$ and Gábor Domokos ${ }^{1}$ \\ Received 10 January 2013; revised 28 August 2013; accepted 4 September 2013; published 10 October 2013.
}

[1] Modeling pebble abrasion during bed load transport is of fundamental importance in fluvial geomorphology, as it may help to understand downstream fining patterns along gravel bed rivers. Here we review a recently published analytical abrasion model called box equations, which can simultaneously track the shape and size evolution of large pebble populations as the cumulative effect of binary collisions between particles. The model predicts that pebble shapes move away from the sphere and develop sharp edges due to collisional abrasion by sand. We present a field study on the downstream evolution of basalt particle shape and size along the Williams River in the Hunter Valley, Australia. Pebbles get flatter and thinner, and several aquafacts (i.e., abraded pebbles with sharp edges) emerge in the downstream reaches, both suggesting the importance of abrasion by sand. Applying box equations with a few fitted parameters, we present a numerical simulation which reproduces both the shape and size evolution of pebbles along the Williams River. The simulation allows tracking of the shape and size evolution of individual particles as well, revealing an interesting phenomenon that particle size controls shape evolution. Box equations, in combination with existing transport concepts, provide a framework for future shape and size evolution studies in sedimentary environments. In particular, they may help to assess the relative importance of size selective transport versus abrasion in causing downstream fining in gravel bed rivers.

Citation: Szabó, T., S. Fityus, and G. Domokos (2013), Abrasion model of downstream changes in grain shape and size along the Williams River, Australia, J. Geophys. Res. Earth Surf., 118, 2059-2071, doi:10.1002/jgrf.20142.

\section{Introduction}

[2] There is a long-standing debate on the relative importance of size selective transport versus abrasion in producing downstream fining in gravel bed rivers [e.g., Brewer et al., 1992; Ferguson et al., 1996; Kodama, 1994; Lewin and Brewer, 2002; Surian, 2002]. Most authors have emphasized sorting by size selective transport as the dominant fining mechanism in various rivers because abrasion intensity was judged to be too small to explain the observed downstream fining rates, especially in cases when the examined part of the river was very short [e.g., Bradley et al., 1972; Dawson, 1988; Ferguson et al., 1996; Seal and Paola, 1995]. This view has been strengthened by the fact that fining rates reported by laboratory abrasion experiments are usually lower than those observed in the field [Lewin and Brewer, 2002].

\footnotetext{
${ }^{1}$ Department of Mechanics, Materials and Structures, Budapest University of Technology and Economics, Budapest, Hungary.

${ }^{2}$ School of Engineering, University of Newcastle, Callaghan, New South Wales, Australia.

Corresponding author: T. Szabó, Department of Mechanics, Materials and Structures, Budapest University of Technology and Economics, Múegyetem rkp. 1-3., K II. 61, Budapest 1111, Hungary. (tszabo@szt.bme.hu)

(C)2013. American Geophysical Union. All Rights Reserved. 2169-9003/13/10.1002/jgrf.20142
}

[3] However, the effectiveness of abrasion in some fluvial environments has also been demonstrated [e.g., Kodama, 1994; Mikos, 1994; Parker, 1991b] and it has been pointed out that abrasion and sorting are not independent because the mobility of particles controls the abrasion rate and vice versa [Jerolmack et al., 2011]. Laboratory abrasion rates may be lower than those observed in the field because various abrasion-in-place processes, e.g., "sandblasting," may play a key role [Brewer et al., 1992; Schumm and Stevens, 1973]. In addition, weathering of clasts can contribute to a higher abrasion rate in natural systems than in experimental conditions [Bradley, 1970] and inappropriate experimental devices can also cause discrepancy between field observations and experiments [Lewin and Brewer, 2002].

[4] Although there are several physical models of downstream fining, most of them consider only size selective transport as the fining process [e.g., Cui et al., 1996; Ferguson et al., 1996; Hoey and Ferguson, 1994, Paola and Seal, 1995]. In recent years, a few pioneering studies managed to physically model the abrasion process during fluvial transport as well [Attal and Lavé, 2009; Chatanantavet et al., 2010; Le Bouteiller et al., 2011; Parker, 1991a]. Some are based on very well documented experimental results coupled with physical considerations [Attal and Lavé, 2009; Le Bouteiller et al., 2011], while others were tested versus field data [Attal and Lavé, 2006; Chatanantavet et al., 2010; Parker, 1991b]. Many of these models rely on the well-known empirical abrasion law by Sternberg [1875], which describes the exponential 
downstream decrease in pebble size observed in many rivers [Surian, 2002; Morris and Williams, 1999].

[5] However, none of the above models describes the changes both in the shape and in the size distribution of the particles during abrasion since they only deal with the size (or alternatively, the mass) of the particles. Here we review a recently published, physically based abrasion model called box equations [Domokos and Gibbons, 2012], which is able to deal with the shape of particles in combination with particle size. Box equations are a heuristic approximation of the geometric partial differential equations (PDEs) governing collisional abrasion [Bloore, 1977; Firey, 1974]. As opposed to the classical partial differential equations of Bloore [1977] and Firey [1974], box equations are a system of coupled ordinary differential equations (ODEs) describing the time evolution of the principle axis ratios as well as the evolution of maximum size. Through direct random simulations, box equations are able to model the shape and size evolution of large pebble populations as the cumulative effect of binary collisions between particles.

[6] There are surprisingly few field studies which examine the evolution of grain size and shape simultaneously in a natural stream [e.g., Bradley et al., 1972; Mikos, 1994; Ueki, 1999], although possible downstream variation in shape may indicate the relative importance of abrasion. In this paper we present a field study performed along the Williams River in the Hunter Valley, Australia. The three principal axis lengths (i.e., the dimensions of the bounding box) of the basalt particles were measured at 12 sites along a $96 \mathrm{~km}$ reach of the Williams River, allowing the axis ratios to be used as shape indices, as has been widely done in accordance with Zingg [1935]. The basalt sediment source is localized in the headwaters, and there is no other basalt source along the river, so the system investigated is undisturbed by additional lateral input between sites. Based on our field observations and box equations, we present a numerical model which reproduces the downstream changes in both the shape and size of gravels along the river.

[7] The structure of the paper is as follows: Section 2 reviews the analytical model (box equations) and its predictions. Section 3 presents a geological and geomorphological background to the study area and discusses the sampling and measuring techniques applied. Section 4 discusses the statistical results on the downstream variation of grain size and shape. The numerical model is presented in section 5 and simulation results, including a detailed parameter study, are presented in section 6 . Finally, the results are summarized in section 7.

\section{Box Equations}

[8] In this section we briefly review a recent theoretical framework called box equations [Domokos and Gibbons, 2012] describing the collective evolution of size and shape in large pebble collections.

\subsection{Deterministic Box Equations}

[9] Box equations are derived from a classical result of Bloore [1977], which is the most general mathematical model describing shape evolution of a single pebble under collisional abrasion. Collisional abrasion refers to the size diminution of a pebble resulting from many binary collisions with other pebbles, and it is distinguished from frictional abrasion which results from the rolling or sliding of a pebble on a substrate. Bloore's partial differential equation (PDE) based on collisional abrasion can be formulated as follows:

$$
v=a(1+2 b H+c K) .
$$

[10] Here $v$ is the speed of abrasion in the inward normal direction at a given point on the particle's surface, and $H$ and $K$ are the mean and Gaussian curvatures at that surface point, respectively. The mean curvature of a given surface point is the average of the two principal curvatures $\kappa_{1}$ and $\kappa_{2}$, while the Gaussian curvature of a given surface point is the product of the principal curvatures, i.e., $H=\left(\kappa_{1}+\kappa_{2}\right) / 2$ and $K=\kappa_{1} \kappa_{2}$. The scalar $a$ defines the attrition speed due to the constant term and by suitable choice of time unit, $a=1$ can be achieved [Domokos and Gibbons, 2012]. In more elaborate models, $a$ could be taken as a function of time. In particular, $a$ may depend on the volume of the particle [Firey, 1974], and later we will use it in this context. In principle, all variables can be written as functions of the scalar distance $R$ from a fixed reference point: $v$ includes time derivative, while $H$ and $K$ include space derivatives of $R$; hence, equation (1) is a PDE. Constants $b$ and $c$ can be computed from the geometry of the abrading particles [Várkonyi and Domokos, 2011] and they are expressed as $b=M / 4 \pi, c=A / 4 \pi$, where $M$ and $A$ denote the integrated mean curvature and surface area of the abraders, respectively. By using constant coefficients $b$ and $c$, we assume that the abraders are identical and invariable in shape and size, that is, $b$ and $c$ in equation (1) do not depend on time. Thus, equation (1) describes collisional abrasion of a single particle in an invariable environment. Very little is known about analytical solutions of Bloore's PDE (1); in particular, it is not known whether it contains nonspherical, nontrivial shapes as stable attractors. Due to the abundance of initial shapes, the global, numerical investigation of equation (1) is still challenging.

[11] In sedimentology, pebble shapes are classically described by the three sizes $L>I>S$ of the bounding box (i.e., the length of the three axes of the approximating triaxial ellipsoid). Based on these dimensions, numerous shape indices have been proposed to describe general particle form [Blott and Pye, 2008]. The simplest and most widely used shape indices, namely the axis ratios $S / L$ and $I / L$, were proposed by Zingg [1935] and Sneed and Folk [1958], to quantify the overall shape of the particles. Throughout this paper, axis ratios $S / L$ and $I / L$ will be denoted by $y_{1}$ and $y_{2}$, respectively, and the semimajor axis $L / 2$ by $y_{3}$.

[12] Box equations are a heuristic approximation of Bloore's equation (1). Instead of tracking the full 3-D geometry of a particle, box equations aim more modestly at tracking the two axis ratios, $y_{1}$ and $y_{2}$, and the semimajor axis, $y_{3}$, of the approximating ellipsoid. While box equations are not a rigorous mathematical approximation of Bloore's PDE (ellipsoids are not invariant under equation (1)), they offer huge conceptual and computational advantages. Whereas equation (1) describes the evolution of a single particle, box equations are based on the concept of mutual abrasion. Their deterministic version describes the interaction between two particles and the stochastic box equations enable the study of the collective evolution of large pebble populations. In the deterministic box equations, the first particle $(\mathbf{y})$ represents the abrading environment for the second particle $(\mathbf{z})$ and vice 


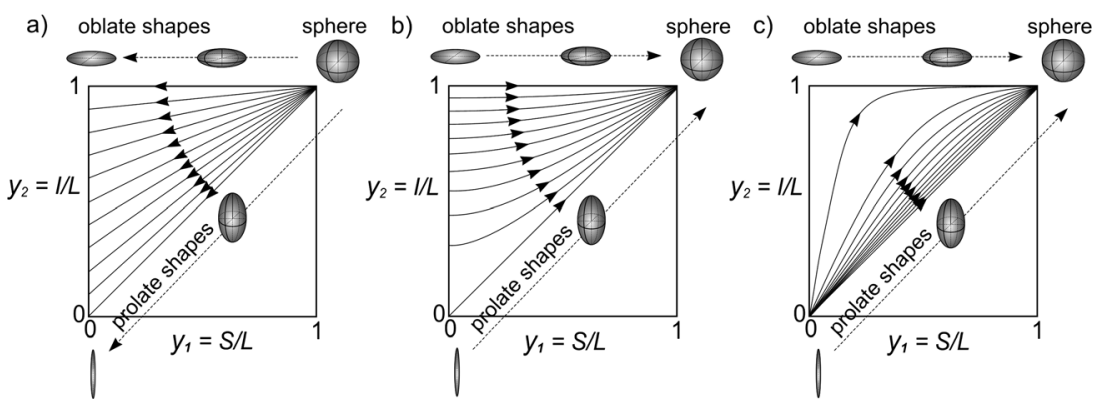

Figure 1. Effect of the three separate terms of box equations based on Domokos and Gibbons [2012]. Assuming that $b$ and $c$ are constants in equations (4)-(5), curves with arrows represent a few selected trajectories of particles (solutions of equations (4)-(5)) on the $y_{1}-y_{2}$ plane. The figures show the effects due to the following: (a) Eikonal term, (b) mean curvature term, and (c) Gaussian curvature term. In Figure 1a, shapes diverge from the sphere, in Figures $1 \mathrm{~b}$ and $1 \mathrm{c}$, shapes approach the sphere. Note that by definition, $y_{2} \geq y_{1}$.

versa, and their mutual interaction is described by a system of coupled ODEs of the following form:

$$
\begin{aligned}
& \frac{\mathrm{d} \mathbf{y}}{\mathrm{d} t}=\dot{\mathbf{y}}=\mathbf{F}(\mathbf{y}, \mathbf{z}) \\
& \frac{\mathrm{d} \mathbf{z}}{\mathrm{d} t}=\dot{\mathbf{z}}=\mathbf{F}(\mathbf{z}, \mathbf{y}) .
\end{aligned}
$$

[13] Here, $\mathbf{y}$ is a three-component vector with components $y_{1}, y_{2}$, and $y_{3}$ and similarly, the axis ratios and the semimajor axis of particle $\mathbf{z}$ is denoted by the vector components $z_{1}, z_{2}$, and $z_{3}$. Using this vector notation, the function $\mathbf{F}$ is also a vector with the three components $F_{1}, F_{2}$, and $F_{3}$, and these component functions can be formulated as follows:

$$
\begin{gathered}
\dot{y}_{i}=F_{i}(\mathbf{y}, \mathbf{z})=\frac{F_{i}^{\mathrm{E}}}{y_{3}}+2 b \frac{F_{i}^{\mathrm{M}}}{y_{3}^{2}}+c \frac{F_{i}^{\mathrm{G}}}{y_{3}^{3}} \text { for } i=1,2, \\
\dot{y}_{3}=F_{3}(\mathbf{y}, \mathbf{z})=-1-\frac{b}{y_{3}} \frac{y_{1}^{2}+y_{2}^{2}}{y_{1}^{2} y_{2}^{2}}-\frac{c}{y_{3}^{2}} \frac{1}{y_{1}^{2} y_{2}^{2}},
\end{gathered}
$$

where

$$
F_{i}^{\mathrm{E}}=y_{i}-1, F_{i}^{\mathrm{M}}=\frac{1-y_{i}^{2}}{2 y_{i}}, F_{i}^{\mathrm{G}}=\frac{1-y_{i}^{3}}{y_{i} y_{j}^{2}}, j=3-i
$$

and

$$
b=\frac{z_{3}\left(z_{1}+z_{2}+1\right)}{3}, c=\frac{z_{3}^{2}\left(z_{1}+z_{2}+z_{1} z_{2}\right)}{3} .
$$

[14] The detailed derivation of box equations (2)-(7) from Bloore's equation (1) can be found in Domokos and Gibbons [2012], where the agreement with Bloore's model is verified analytically, numerically, and experimentally. In this study we only consider collisional abrasion; however, frictional abrasion can also be included as simple additive terms in box equations [Domokos and Gibbons, 2012].

[15] Both the original Bloore equation (1) and its box approximation (equations (2)-(7)) consist of the linear combination of three terms to which direct physical and geological interpretation can be attached. The first constant term (the unit constant) in equation (1) is called the Eikonal term. The second term $(2 b H)$ and the third term $(c K)$ are referred to as the mean curvature and the Gaussian curvature term, respectively. Similarly, in case of box equations, the superscripts $E, M$ and $G$ in the three terms in equation (4) stand for Eikonal, mean curvature and Gaussian curvature, respectively. The effect of the three individual terms of box equations can be globally illustrated in the $y_{1}-y_{2}$ plane (Figure 1 ). We can see that the Eikonal term is driving shapes away from the sphere while the two curvature terms do the opposite. The same behavior can be observed in the original Bloore PDE (1) as well; it was proven by Firey [1974] and Huisken [1984] that under the effect of the curvature terms, initial shapes converge to the sphere (for illustration, see Figure 2b). The Eikonal term dominates abrasion if the abrading particles are relatively small with respect to the abraded particle. This is most readily seen in case of spherical particles: if the abraded particle has radius $R$ and the abrading particles have radius $r$ then the mean curvature and Gaussian curvature terms are constant multiples of $r / R$ and $(r / R)^{2}$, respectively [Domokos and Gibbons, http://arxiv.org/abs/1307.5633, 2013]. Therefore, if $r / R$ is small, the curvature-driven terms become negligible compared to the Eikonal term. Similarly, the mean and Gaussian curvature terms dominate abrasion in case of relatively large abraders (large $r / R$ ). In case of nonspherical particles the situation is qualitatively similar [Domokos and Gibbons, 2012; Várkonyi and Domokos, 2011]. The detailed geometry of the Eikonal term is of particular interest [Arnold, 1986] as it accounts for the formation of sharp edges and planar areas on the abraded particle (Figure 2a). This was shown in case of asteroids [Domokos et al., 2009] and for the formation of ventifact shapes by wind-blown sand in deserts [Knight, 2008; Várkonyi and Laity, 2012].

\subsection{Stochastic Box Equations}

[16] In the stochastic interpretation, box equations generate a discrete, random Markov process [Domokos and Gibbons, 2012] describing the size and shape evolution of a whole particle population as the cumulative effect of binary collisions between particles. In this approach, $\mathbf{y}$ and $\mathbf{z}$ are random variables with identical distributions, i.e., we consider $N$ particles from which we choose $\mathbf{y}$ and $\mathbf{z}$ randomly, 

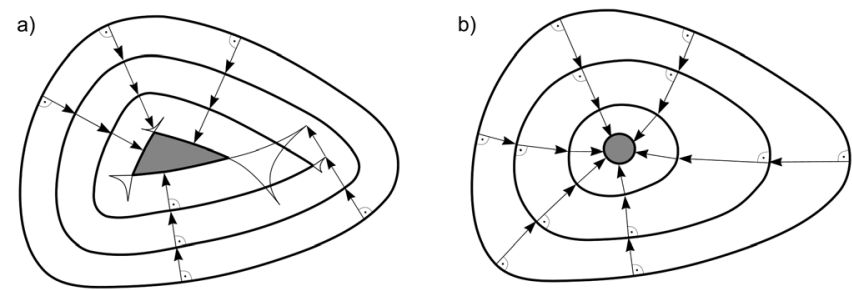

Figure 2. Illustration of the evolution of a two-dimensional pebble shape under the separate effect of (a) the Eikonal term and (b) the curvature term. Note that in two dimensions, there is only one curvature instead of the mean and Gaussian curvatures in three dimensions. Arrows indicate the approximate path of a few selected surface points during abrasion. Observe that in Figure 2a, typical initial shapes develop cusps and self-intersections. The latter appear as vertices (in three dimensions, sharp edges) on the physically existing solution (shaded part). The curvature term (Figure 2b) drives the original shape toward a circle (in three dimensions, a sphere).

and run the straightforward discretization of equation (2)-(3) for a very short time period $\Delta t$, to obtain the updated data $\left(\mathbf{y}^{i+1}\right.$ and $\left.\mathbf{z}^{i+1}\right)$ from the original status $\left(\mathbf{y}^{\mathrm{i}}\right.$ and $\left.\mathbf{z}^{\mathrm{i}}\right)$ :

$$
\begin{aligned}
& \mathbf{y}^{i+1}=\mathbf{y}^{i}+\Delta t \cdot \mathbf{F}\left(\mathbf{y}^{i}, \mathbf{z}^{i}\right) \\
& \mathbf{z}^{i+1}=\mathbf{z}^{i}+\Delta t \cdot \mathbf{F}\left(\mathbf{z}^{i}, \mathbf{y}^{i}\right) .
\end{aligned}
$$

[17] Such an iterative step is an averaged, summarized result of several binary collisions between the two selected particles. As discussed in section 5.2.2 in more detail, the random draw can be either uncorrelated, or correlated by shape and/or size, modeling the effect of segregation. We assume that a particle $\mathbf{y}$ exits from the population if $y_{3}<0$ (i.e., its size vanishes), and in the rare event that its axis ratio leaves the meaningful region (i.e., when one of the following holds: $\left.y_{1}<0, y_{2}<0, y_{1}>1, y_{2}>1\right)$. The latter can only happen due to the discretization, just before size vanishes. When $N$ approaches infinity, equations (8)-(9) define the PDE describing the evolution of the density functions of $y_{1}$, $y_{2}$, and $y_{3}$; i.e., (8)-(9) offer a full statistical description of the evolution of axis ratios and particle size. The numerical simulation of (8)-(9) is very convenient; on a laptop a few hundred particles can be easily tracked both statistically and individually.

\subsection{Fragment Production}

[18] The original box equations (equations (2)-(7)) can be modified to deal with the production of fine particles during binary collisions. This is done by adding the abraded material to the particle population to make the model more realistic. Fragment production has a direct effect on the equations which are introduced in a simplistic model below. To be able to treat the production of fine particles analytically, some reasonable assumptions are needed for the geometry and energy of fragmentation. Although these assumptions have not yet been verified by any field or experimental data, they enable the model to be kept as transparent and simple as possible.

[19] The first assumption is that in every iterative step (averaged collision) the same number of new small particles appears. Instead of this, other assumptions could also be adopted, e.g., that the new particles are identical in shape and size. In this latter case, the geometry of the new particles can be considered as it corresponds to the median/mean value of the true fragment distribution. The second assumption is that particles have a constant collisional velocity. In this way the collisional energy $E_{c}$ is proportional to the combined mass $\left(m_{y}+m_{z}\right)$ of the two colliding particles: $E_{c} \propto m_{y}+m_{z}$. Although the collisional velocity scales linearly with fluid velocity [Attal and Lavé, 2009; Lajeunesse, et al., 2010] in the case of full transport, and fluid velocity typically increases in the downstream direction along a river, this change is approximately proportional to the $0.1-0.2$ power of the discharge [Leopold, 1953; Carlston, 1969]. Thus, velocity appears to change much more slowly downstream than particle mass. Accordingly, for simplicity, this effect is neglected here. However, if the velocity profile for the whole river is known, then it can be directly implemented into a numerical simulation.

[20] Now we consider fragment production, i.e., the abraded material $\left(\Delta m_{y}+\Delta m_{z}\right)$ breaks up into smaller pieces which are initially in contact with each other before separating along fissures at a contact surface $S$. We assume that the energy $E_{b}$ needed for the breakup is proportional to this contact surface [Griffith, 1921]. Because the same number of new particles appears in every binary collision, $S$ is proportional to $\left(\Delta m_{y}+\Delta m_{z}\right)^{2 / 3}$ (since the linear length of the abraded material scales with $\left.\left(\Delta m_{y}+\Delta m_{z}\right)^{1 / 3}\right)$. Thus, we have $E_{b} \propto S \propto\left(\Delta m_{y}+\Delta m_{z}\right)^{2 / 3}$. If the ratio of collisional energy to fragmentation energy depends neither on time nor on particle size, we obtain $\left(\Delta m_{y}+\Delta m_{z}\right) \propto\left(m_{y}+m_{z}\right)^{3 / 2}$. The linear length change for pebble $\mathbf{y}$ is approximately $\left(\Delta m_{y}\right)^{1 / 3}$, and from the previous equation we have $\left(\Delta m_{y}\right)^{1 / 3} \propto\left(m_{y}+m_{z}\right)^{1 / 2}$. Clearly, the same holds for pebble $\mathbf{z}$ as well. From this, we obtain that $\dot{\mathbf{y}}, \dot{\mathbf{z}} \propto\left(m_{y}+m_{z}\right)^{I / 2}$. Then, by combining it with equations (2)-(3), we have

$$
\begin{aligned}
& \dot{\mathbf{y}}=\mathbf{F}(\mathbf{y}, \mathbf{z}) \cdot C(\mathbf{y}, \mathbf{z}) \\
& \dot{\mathbf{z}}=\mathbf{F}(\mathbf{z}, \mathbf{y}) \cdot C(\mathbf{z}, \mathbf{y}),
\end{aligned}
$$

where $C$ is a dimensionless multiplier and its magnitude is given by $C(\mathbf{y}, \mathbf{z})=C(\mathbf{z}, \mathbf{y})=\left(m_{y}+m_{z}\right)^{1 / 2}$. A very similar mass multiplier has already been proposed by Firey [1974].

[21] Although this is just a simplistic model, $C$ has an important effect on the mass evolution of particles. In the case of two particles, the shape of the trajectories in the $y_{1}-y_{2}-y_{3}$ and $z_{1}-z_{2}-z_{3}$ space do not change compared to equations (2)-(3) since $C$ is a symmetric multiplier $(C(\mathbf{y}, \mathbf{z})=C(\mathbf{z}, \mathbf{y}))$ and operates on each of the three scalar equations corresponding to the three vector components of $\mathbf{y}$ and $\mathbf{z}$. However, the velocity 
SZABÓ ET AL.: PEBBLE SHAPE EVOLUTION, WILLIAMS RIVER

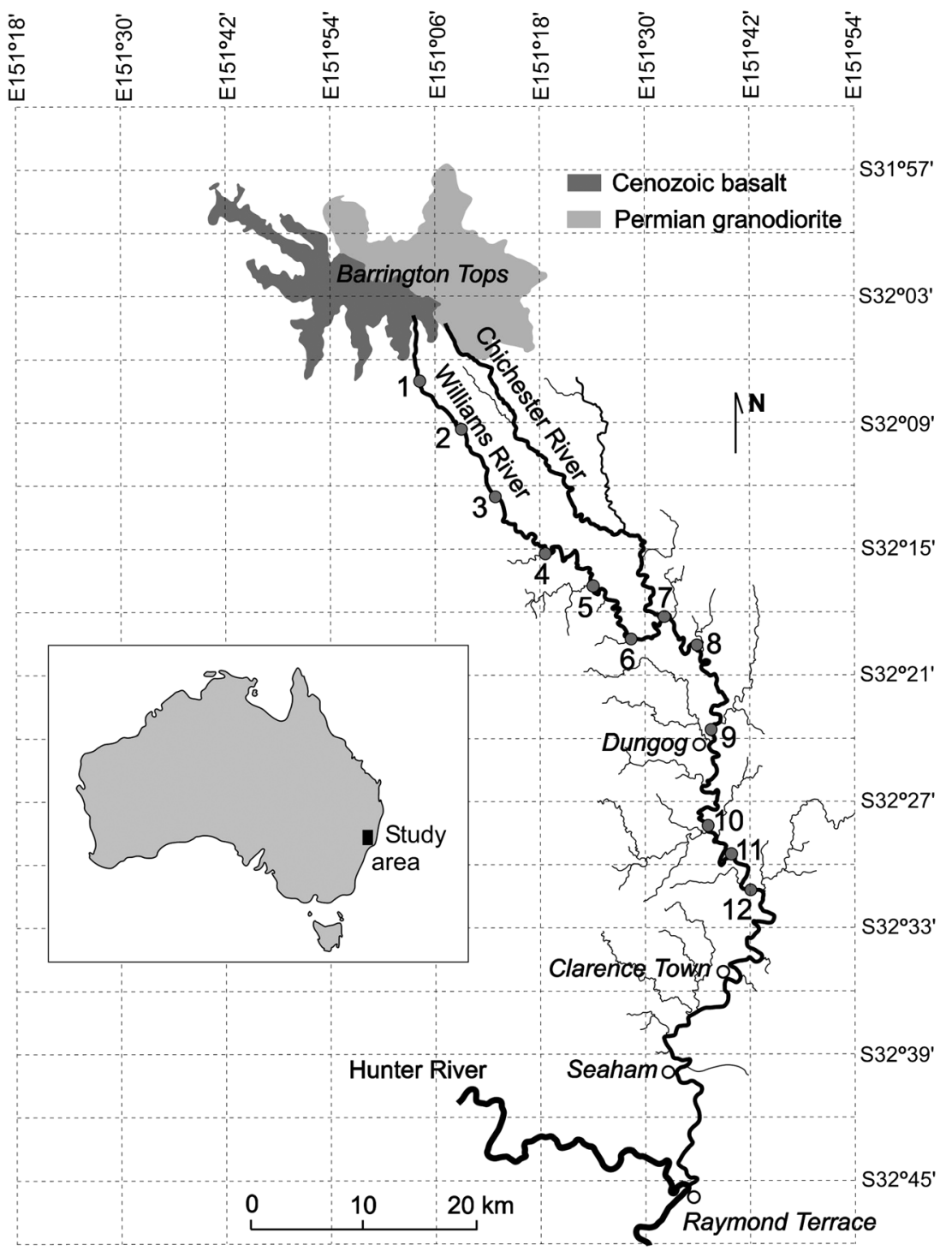

Figure 3. Study area and the 12 sites.

along these trajectories will be modified by applying $C$ since $m_{y}$ and $m_{z}$ depend on time.

[22] More generally, $C$ can be defined separately for particle $\mathbf{y}$ and $\mathbf{z}$ as $C_{y}(\mathbf{y}, \mathbf{z})=a_{y}\left(m_{y}+m_{z}\right)^{1 / 2}$ and $C_{z}(\mathbf{z}, \mathbf{y})=$ $a_{z}\left(m_{y}+m_{z}\right)^{1 / 2}$, where constants $a_{y}$ and $a_{z}$ represent the durability of particle $\mathbf{y}$ and $\mathbf{z}$. In this way, the equations are able to model the effect that abrasion rate is strongly controlled by lithology. Such a constant was already applied by Bloore [1977] in his equation. In the present study, we track only basalt particles along the Williams River, thus in our case, $a_{y}=a_{z}$. By applying the same argument as in section 2.2, equations (10)-(11) can be discretized to be able to follow the evolution of a whole particle population:

$$
\begin{aligned}
& \mathbf{y}^{i+1}=\mathbf{y}^{i}+\Delta t \cdot \mathbf{F}\left(\mathbf{y}^{i}, \mathbf{z}^{i}\right) \cdot C_{y}\left(a_{y}, \mathbf{y}^{i}, \mathbf{z}^{i}\right) \\
& \mathbf{z}^{i+1}=\mathbf{z}^{i}+\Delta t \cdot \mathbf{F}\left(\mathbf{z}^{i}, \mathbf{y}^{i}\right) \cdot C_{z}\left(a_{z}, \mathbf{z}^{i}, \mathbf{y}^{i}\right) .
\end{aligned}
$$

[23] Equations (12)-(13) will be applied in the numerical simulation in section 5 .

\section{Study Area and Sampling Methods}

\subsection{Study Area}

[24] The $148 \mathrm{~km}$ long Williams River is located in the Hunter Valley, New South Wales, Australia (Figure 3), draining a catchment of more than $1000 \mathrm{~km}^{2}$ [Erskine, 2001]. The headwaters of the Williams River are on the Barrington Plateau at an elevation of around $1100 \mathrm{~m}$ (Figure 4). The river flows to the south/southeast until it joins the Hunter River at Raymond Terrace. The Barrington Plateau is a geomorphic

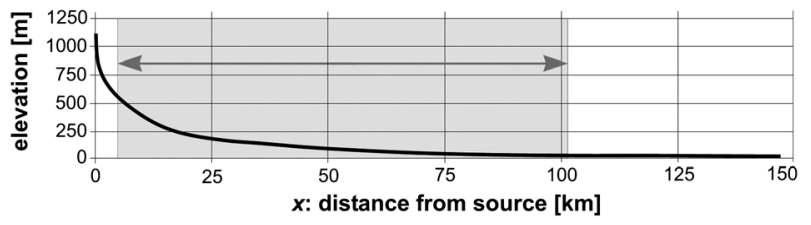

Figure 4. Approximate longitudinal river profile based on Google Earth. The shaded part indicates the examined part of the river, where gravel beds dominate. 
Table 1. Estimation of the Starting and Ending Sample Sizes in the Numerical Model ${ }^{\mathrm{a}}$

\begin{tabular}{lcc}
\hline Site & Site 1 & Site 12 \\
\hline Sample size & 141 & 100 \\
Area size & $1.2 \cdot 1.5=1.8 \mathrm{~m}^{2}$ & $2.5 \cdot 2.5=6.25 \mathrm{~m}^{2}$ \\
Expected sample size in $6.25 \mathrm{~m}^{2}$ & $\sim 490$ & 100 \\
\hline
\end{tabular}

${ }^{\mathrm{a} S}$ Sample sizes refer to particles larger than $20 \mathrm{~mm}$.

feature derived from the Cenozoic-aged Barrington Shield Volcano [Sutherland and Fanning, 2001], and consequently, the first $5 \mathrm{~km}$ of the river is incised into basalt rocks as the river drops through the escarpment of the plateau. This is the only significant source of basalt detritus anywhere along the river, with the rest of its length passing through sedimentary and felsic volcanic rocks of Carboniferous age. The Williams River is unaffected by significant anthropogenic interventions such as dams which would alter sediment dynamics. Its only substantial tributary, the Chichester River, has its headwaters in a geologically different part of the mountains, in the Permian-aged Barrington Tops Granodiorite pluton [Sutherland and Fanning, 2001]. The Williams River was selected for this study because it provided the opportunity to study the downstream evolution of basalt particle shape and size, in a sample of material which has traveled a known distance from its localized point source. Basalt itself has favorable properties: it has a characteristic appearance allowing it to be positively identified in a mixed sample and it is relatively homogeneous and isotropic, so shape evolution is not strongly affected by a directional fabric such as in rocks with sedimentary bedding planes or foliation.

[25] Throughout the study reach, which extends from $6 \mathrm{~km}$ to $102 \mathrm{~km}$ from its source, the river has a gravel bed and large mobile gravel bars. In the upper $15 \mathrm{~km}$ of the study reach, the river runs in a classical, narrow $\mathrm{V}$-shaped valley. Below this, the gradient decreases considerably (Figure 4) and the river starts to meander. Downstream of the study reach, in the last $50 \mathrm{~km}$, the river becomes deeper and wider and no gravel bars can be found: the submerged river bed is dominated by sands. This phenomenon is called gravel-sand transition and has been documented in many other downstream-fining studies [e.g., Ferguson, 2003; Frings, 2011; Jerolmack and Brzinski, 2010].

\subsection{Sampling and Measuring Methods}

[26] Basalt particles larger than $20 \mathrm{~mm}$ were collected from the surface layer of gravel bars at 12 sites along the study reach. The localities were approximately regularly spaced with an average spacing of about $9 \mathrm{~km}$ (Figure 3). Where possible, gravel samples were taken consistently at the center of the bars in order to reduce bias due to local variations of grain size within a bar with large-scale downstream fining. Since only the basalt particles were tracked, classical grid-based sampling methods like Wolman pebble count [Wolman, 1954] were inappropriate to collect the samples. Therefore, we framed a nominal rectangular area and collected every basalt particle from the surface within that area. Although gravel bar material shows an apparent surface coarsening in many gravel bed rivers [Attal and Lavé, 2006], our sampling focused only on the top sediment layer. The size of the rectangular area was chosen to yield a sample of between 100-200 basalt particles. The size of the required rectangle increased with increasing distance downstream as the relative frequency of basalt fragments larger than $20 \mathrm{~mm}$ decreased. Data from sites 1 and 12 are presented as an example in Table 1. The average sample size was 140 basalt particles.

[27] Sizes of the bounding box (i.e., the three axes where $L>I>S$ ) of each particle were measured, using measuring frames for boulders and cobbles and a digital caliper for pebbles. From these, axis ratios $y_{1}=S / L$ and $y_{2}=I / L$, as well as the semimajor axis $y_{3}=L / 2$ were computed.

\section{Field Results and Field Observations}

\subsection{Field Results}

[28] Field results are shown in Figure 5, where all variables $\left(y_{1}, y_{2}, y_{3}\right)$ are plotted against the distance from source $(x)$. Axis ratios $y_{1}, y_{2}$ are nearly constant in the first part of the river; however, there is a slight decrease in the lower reaches. Since the seminal paper of Sternberg [1875], the following exponential law has been adopted to describe the downstream decrease of particle size (or alternatively, particle mass) in many rivers [Morris and Williams, 1999]:

$$
y_{3}(x)=y_{3}^{0} \cdot e^{-\alpha \cdot x},
$$

where $y_{3}^{0}$ is the semimajor axis at the source of the river, $x$ is the distance from the source and $\alpha$ is the so-called diminution coefficient. Application of Sternberg's classical equation for downstream fining allows the exponent in (14) to be approximated for the Williams River and compared to the fining rates of other natural streams.

\subsection{Interpretation of the Field Results and Field Observations}

\subsubsection{Downstream Decrease in Size}

[29] By using the substantial database in the literature [Surian, 2002; Morris and Williams, 1999], the diminution coefficient $\alpha$ (cf., Sternberg's law (14)) observed in the Williams River can be directly compared to the exponents found in other natural streams. Although the diminution coefficient corresponding to the mean pebble size may be underestimated in our measurements because sampling excluded sediment finer than $20 \mathrm{~mm}$, the $\alpha$ value based on the maximal size can be compared to the corresponding maximum size exponents [Morris and Williams, 1999]. The diminution coefficient for the maximal size found for the Williams River is relatively small $\left(\alpha=0.018 \mathrm{~km}^{-1}\right)$, which suggests that abrasion alone may produce the observed downstream fining rate. This hypothesis is in agreement with reported laboratory experiments which demonstrate that volcanic rocks could produce abrasion rates in this range [Attal and Lavé, 2009; Lewin and Brewer, 2002; Morris and Williams, 1999].

\subsubsection{Axis Ratios and Shape}

[30] Changes in pebble shape along the river are indicators of the importance of abrasion. As presented in section 2, in the presence of sand, pebbles typically get flatter and/or thinner (oblate and prolate, Figure 1a). The observed slight decrease in the axis ratios along the Williams River reflects this process, i.e., it indicates that sandblasting by the overpassing suspended load becomes important in the lower part of the river.

[31] This hypothesis is strengthened by two field observations. First, as mentioned in section 3, the amount of sand 

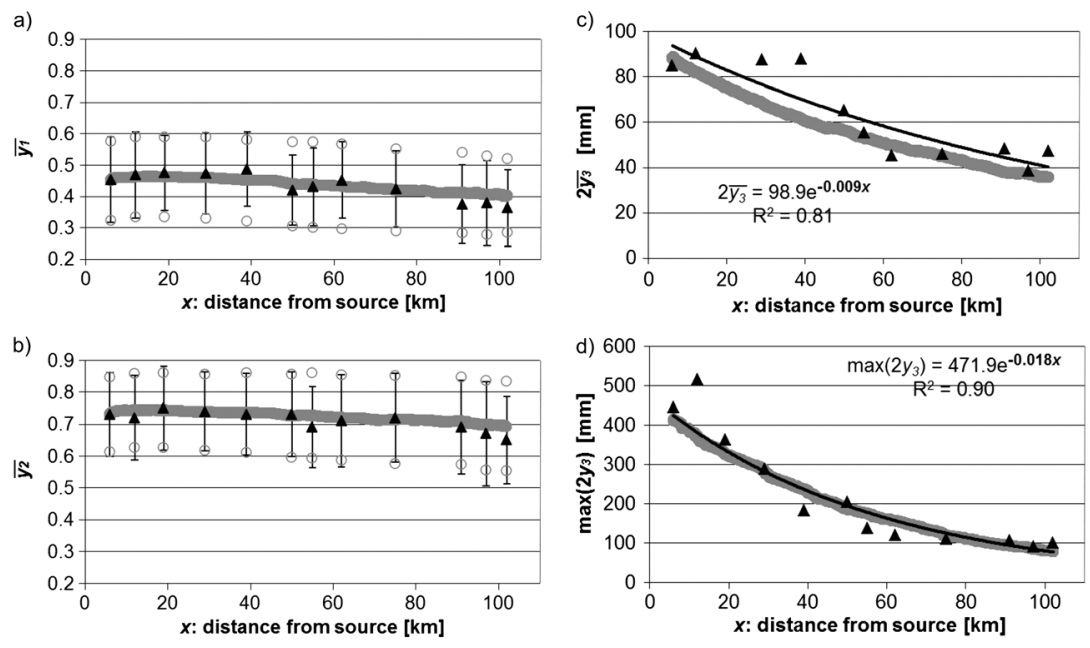

Figure 5. Field results and simulation results. (a) Mean value of axis ratio $y_{1}$ (axis ratio $S / L$ ). (b) Mean value of axis ratio $y_{2}$ (axis ratio $I / L$ ). (c) Mean value of particle size $2 y_{3}$ (axis length $L$ ). (d) Maximum value of particle size $2 y_{3}$, where maxima are computed as the average of the five largest particles. Note that sizes are multiplied by 2 because $y_{3}$ denotes the semimajor axis. Model results are given at $\Delta t=1 / 1000, n=1000$, $m=100,000$. All variables are plotted against $x$, the distance from the source of the river. Field results are represented by black triangles, while model results are shown by thick gray lines. In Figures $5 \mathrm{a}$ and $5 \mathrm{~b}$, the standard deviation of the field results is also indicated, the corresponding model results are represented by gray open circles. In Figures 5c and 5d, black trendlines, exponential equations, and the squares of correlation coefficients are also presented.

significantly increases with increasing distance downstream, especially in the lowest three sites. This phenomenon is usually referred to as gravel-sand transition [Ferguson, 2003; Frings, 2011; Jerolmack and Brzinski, 2010]. Second, in the lower tracts of the river, at the lowest three sites, we found a few aquafact shapes [Kuenen, 1947], i.e., pebbles having sharp edges and planar faces (Figure 6). These shapes are very similar to those of ventifacts formed by wind-blown sand [Knight, 2008] (specifically, to those of einkanters, zweikanters, and dreikanters [Greeley et al., 2002]) but are called aquafacts since here they occur in a fluvial environment [Kuenen, 1947]. Although the occurrence of aquafacts in river and coastal environments was established long ago [Kuenen, 1947; Frankel, 1955; Taljaard, 1939], so far, minimal attention has been given to them. In section 2 we showed that Bloore's equation (1) predicts the emergence of these shapes in situations of continual collisions with relatively small particles. The quantitative characterization of the abundance versus rarity of aquafacts at a given place would be a very hard task since there is no objective measure which would help to distinguish between aquafacts and nonaquafacts. However, the emergence of several, extremely characteristic shapes in the lower reaches qualitatively indicates that the sandblasting process observed already by Brewer et al. [1992] is occurring in the Williams River, i.e., these shapes are formed by the interactions with the overpassing suspended load. In the lower part of the Williams River, river bars are partly stabilized by vegetation which may facilitate the formation of aquafacts, since pebbles become less mobile and the abrasion effect of the overpassing suspended sediment can start to develop sharp edges on the pebbles.

[32] It is important to point out that there is no consensus among researchers as to whether sand particles are effective in abrading larger grains under water. While some researchers argue that collisions are completely damped by the viscous pressure that the water exerts on the particles when they approach each other [Schmeeckle et al., 2001], others emphasize the importance of suspended sediment because of the heightened impact velocity of small particles due to turbulence [Lamb et al., 2008]. Here it is assumed that small particles are efficient in causing abrasion; however, it remains an important and open question which calls for thorough laboratory experiments in the future.

\section{Numerical Simulation}

[33] Based on the field observations and data discussed in the previous section, we assume that abrasion is the dominant

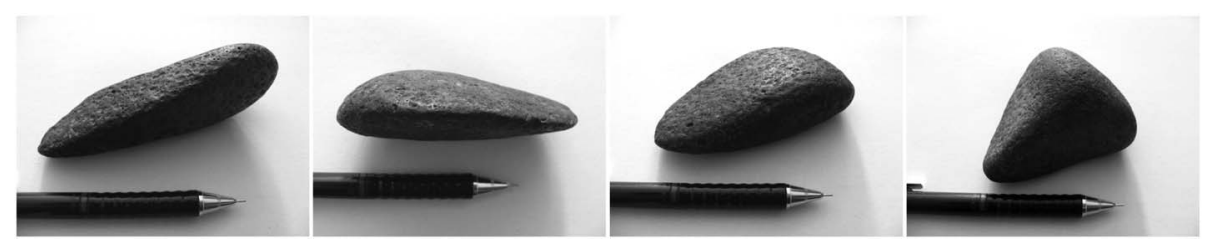

Figure 6. Aquafacts [Kuenen, 1947] found in the lower part of the Williams River. Observe the sharp edges and flat faces. 

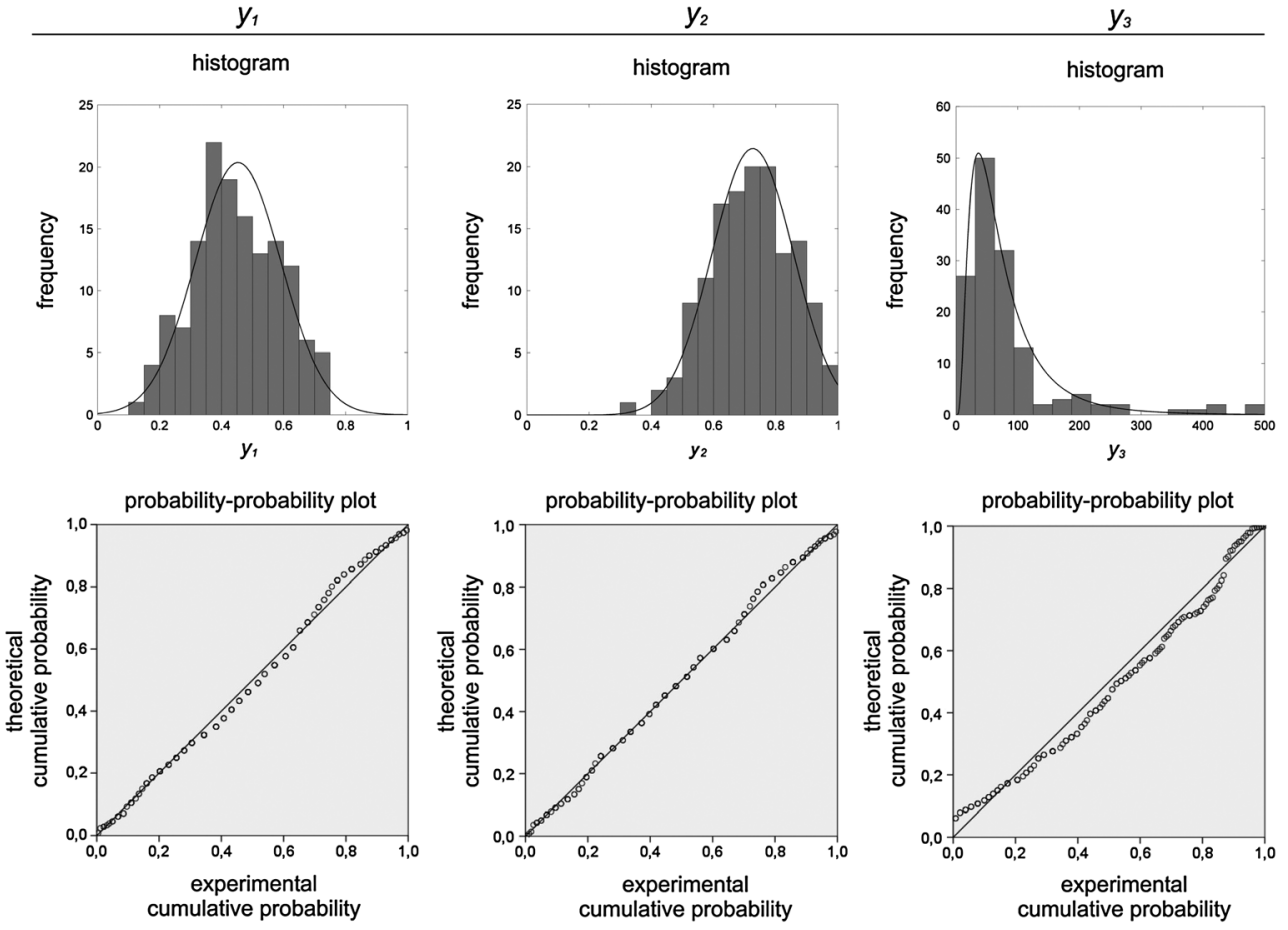

Figure 7. Shape and size distributions at site 1. The $\mu$ and $\sigma$ denote mean and standard deviation, respectively. Axis ratio $y_{1}$ (left column) is approximated with a normal distribution with parameters $\mu=0.453$ and $\sigma=0.138$, axis ratio $y_{2}$ (middle column) with a normal distribution with parameters $\mu=0.726$ and $\sigma=0.131$, and size $y_{3}$ (right column) with a lognormal distribution with parameters $\mu=4.122=\ln (61.702)$ and $\sigma=0.729$. Observe the very good agreement in the probability-probability plots.

process in causing downstream fining along the Williams River. Relying on this hypothesis, we present a numerical abrasion model, relying on box equations with fragment production (equations (12)-(13)), to explain downstream fining and shape variation along the Williams River.

\subsection{Input Data and Sample Sizes}

[34] The numerical model simulates the downstream changes in the basalt particle population of the Williams River, using the observed shape and size distributions of site 1 as a starting point. Then, from this initial condition, the evolution of the distributions can be tracked in the model and compared with the field results at the remaining 11 sites. Axis ratios $y_{1}$ and $y_{2}$ follow normal distributions along the whole river. Size distributions $\left(y_{3}\right)$ can be best approximated by lognormal distributions, as has been found in other sedimentary environments [Blott and Pye, 2001; Fityus et al., 2013]. Histograms and fitted probability distributions for site 1 are presented in Figure 7. Using these theoretical distributions, a random initial sample with an arbitrary number of particles can be generated.

[35] Section 3.2 noted that only basalt particles larger than $20 \mathrm{~mm}$ were collected. We have also considered input data on sample size, and in particular, the change in the relative frequency of particles larger than $20 \mathrm{~mm}$ from sites 1 to 12 . Table 1 shows that at site 1, 141 particles larger than $20 \mathrm{~mm}$ were found in an area of $1.8 \mathrm{~m}^{2}$. If all of these particles were to survive without abrasion, then the maximum number of particles expected in the $6.25 \mathrm{~m}^{2}$ sample area at site 12 would be 490 . However, as shown in Table 1, exactly 100 particles larger than $20 \mathrm{~mm}$ were found in the $6.25 \mathrm{~m}^{2}$ sample area at site 12 . Therefore, assuming that the additional nonbasalt sediment supply from the banks and tributaries can be neglected, and that there has not been any size selective deposition along the reach, we started the simulation with 490 particles larger than $20 \mathrm{~mm}$, and stopped it when 100 particles that were larger than $20 \mathrm{~mm}$ remained. Needless to say, it does not mean that this is the smallest size in the simulated particle population, as we will see it in the next subsection.

\subsection{Model Assumptions and Parameters}

[36] To be able to compare simulation results to field results, we assume a linear connection between model time and the distance from the source. In other words, we assume that all particles travel at the same speed, regardless of their size. This assumption is not a restriction arising from equations (12)-(13); in future work this assumption could be removed by coupling our model with existing transport concepts. Due to the assumed linear connection and the facts that the starting and ending sample sizes are fixed and we track only one lithology in the model, the results do not change by assuming $a_{y}=a_{z}=1$ in equations (12)-(13).

[37] To describe the most essential features of the abrasion process in the Williams River, some model parameters are needed. The observed downstream changes in the Williams River can be reconstructed with two fitted parameters based on physical assumptions. The first parameter is connected to the increasing amount of sand noticed along the river. The second parameter models the effect of particle segregation by size, which can be considered as a possible consequence of size selective transport on the abrasion process. 


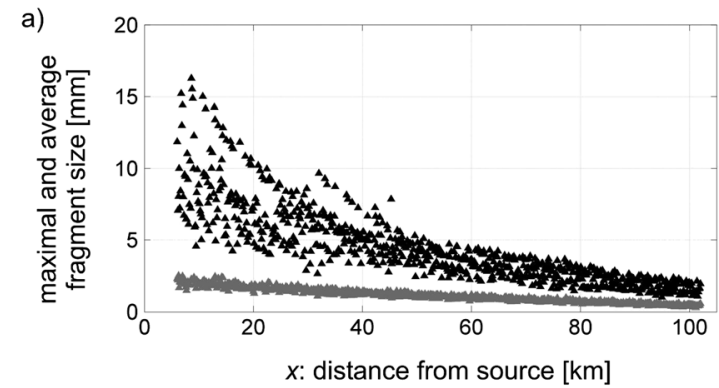

b)
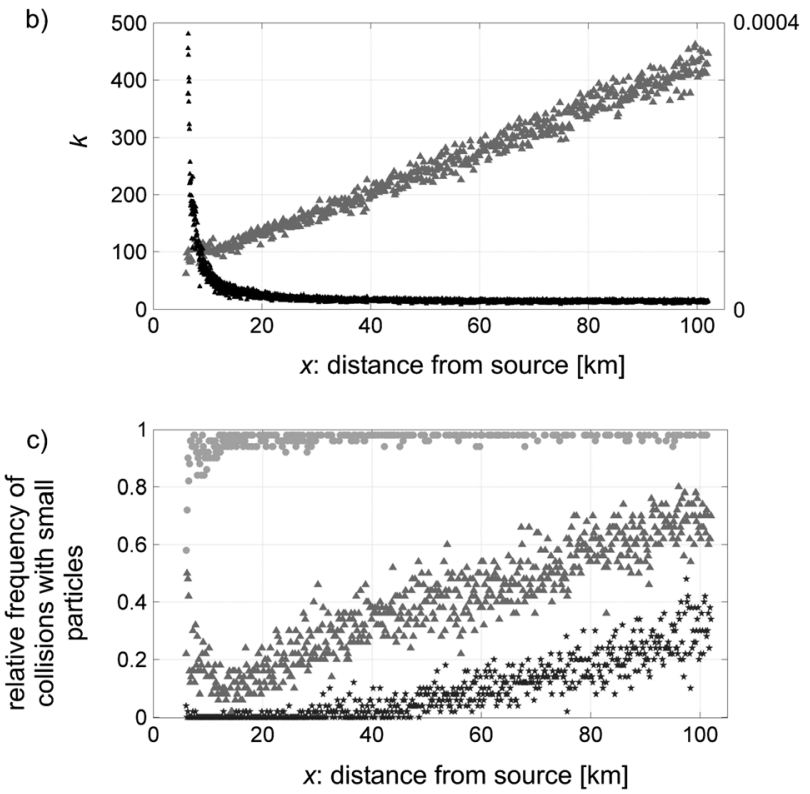

Figure 8. The effect of model parameters in the simulation. $n=1000, \Delta t=1 / 1000, m=100,000$. Data points are computed as an average of 50 consecutive iterative steps. (a) Maximum (black) and average (gray) fragment size appearing in the particle population. (b) Left axis/gray points: The number of potential $z$ particles, $k$ (see explanation in the text). Right axis/black points: the ratio $k / N$, where $N$ is the total number of particles. $k / N$ indicates the effectiveness of segregation by size. (c) The relative frequency of collisions with particles smaller than: $16 \mathrm{~mm}$ (light gray circles), $8 \mathrm{~mm}$ (gray triangles) and $4 \mathrm{~mm}$ (black stars).

The effects of these parameters and the sensitivity of the model will be assessed in detail in section 6 .

\subsubsection{Sand Production}

[38] As described in section 2.3, our model accounts for fragment production by assuming that in each (averaged) binary collision the same number of new fragments appears, which leads to equations (10)-(11). Specifically, in every iterative step, $n$ small particles are produced, both for particle $\mathbf{y}$ and particle $\mathbf{z}$, so $n$ is the first parameter in the numerical simulation. In the following, we use the value $n=1000$ obtained from fitting the model to the field data. At first sight $n$ may seem to be overestimated, but we emphasize that one iterative step is the averaged, cumulative effect of many binary collisions. In addition, we assume that the new particles are spherical and that they do not get abraded any more. These are convenient simplifications which substantially help to reduce execution time (since in this way, the $n$ particles produced in one iterative step remain identical and can be stored in one vector). The effects of these assumptions on the numerical results are negligible, as is outlined below.

[39] The first assumption (spherical particles) modifies the true shape distribution of new small fragments; however, this has almost no effect on the shape and size evolution of the much bigger pebble-, cobble-, and boulder-sized particles, which are tracked in the model and were collected in the field. When a pebble- to boulder-sized particle $\mathbf{y}$ collides with a sand-sized particle $\mathbf{z}$, then $z_{3}<<y_{3}$. Thus, based on equations (4)-(7), the first (Eikonal) term of equations (4)-(5) will dominate. However, the Eikonal term itself does not include the shape of particle $\mathbf{z}$. Nevertheless, well-controlled laboratory experiments would be needed to obtain not only the size distribution but also the shape distribution of fragments produced in collisions for different rock types. If these data were available, they could be directly applied in the numerical model to be able to track the shape and size evolution of the full particle range, not only particles larger than sands. Since we have no field data on sands, this was not our current objective.

[40] Nonerodible new fragments are not a critical assumption because the number of new particles is three orders of magnitude larger than the number of iterative steps (since $n=1000$ new particles appear in each iterative step), so even if some new particles would disappear due to wear in the binary collisions, their total number would change only slightly. In addition, the compressive model of Kendall [1978] shows that the energy needed to damage very small grains tends to infinity as grains become smaller.

[41] Clearly, the average size of the fragments in the model depends on the time step $\Delta t$ as well because the linear length change is proportional to $\Delta t$. Thus, $\Delta t$ is not an independent parameter and has to be set to a sufficiently small value to reach a reasonable approximation of the true solutions of box equations. Then, $n$ can be treated as the free parameter. In our case $\Delta t=1 / 1000$, which induces a small volume loss in each iterative step.

\subsubsection{Segregation by Size}

[42] We introduce a second effect in the model, which is the possibility that particles segregate by size during their transport in the river, thus particles are more likely to collide with particles of similar size. Segregation by size has a direct effect on the abrasion process itself. This phenomenon may be considered as the possible influence that size selective transport has on abrasion.

[43] Segregation by size is modeled in the following way. For every iterative step, first, a particle $\mathbf{y}$ is randomly (with equal probability) chosen from the particle population. Second, $k$ potential $\mathbf{z}$ particles are chosen, again randomly from the whole particle population (i.e., newly produced fragments are also included). Then, from these $k$ particles, the particle which is closest in size compared to particle $\mathbf{y}$ is chosen, i.e., the particle $\mathbf{z}$ where $\left|z_{3}-y_{3}\right|$ is minimal. Thus, in this way the model favors particle collision between particles that are nearer in size, and $k$ is proportional to the intensity of segregation. After particle $\mathbf{y}$ and $\mathbf{z}$ are chosen, equations (12)-(13) with $a_{y}=a_{z}=1$ are applied to them. The number of possible $\mathbf{z}$ particles, $k$, is determined in such a way that it always corresponds to the $1 / \mathrm{m}$ part of the total volume $V_{\text {total }}$ of the whole population, where constant $m$ is the second parameter. In our 
a)

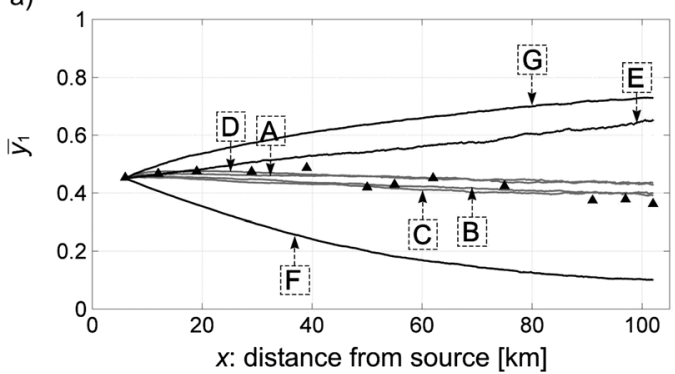

b)

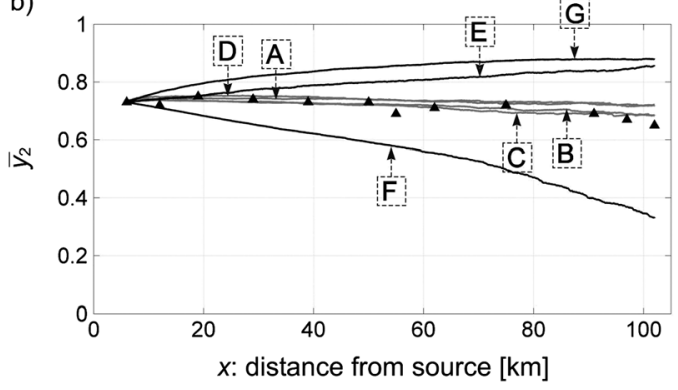

c)

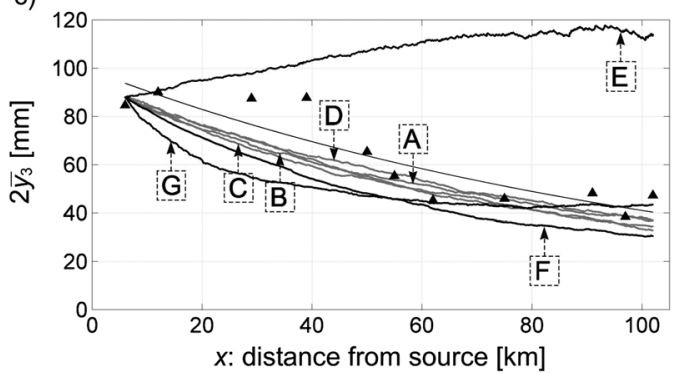

d)

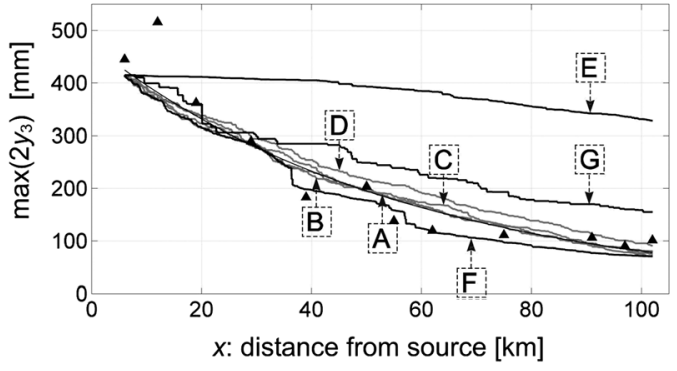

Figure 9. Parameter sensitivity study. The investigated variables are the same as those of Figure 5; field data are represented by black triangles; $\Delta t=1 / 1000$. Gray lines: Case A: $n=500, m=100,000 ;$ Case B: $n=2000, m=100,000$; Case C: $n=1000, m=200,000$; Case D: $n=1000, m=50,000$. Black lines: Case $\mathrm{E}$ : no fragment production, no segregation, $n=0, k=1$; Case $\mathrm{F}$ : fragment production with no segregation, $n=1000, k=1$; Case G: fragment production with strong segregation, $n=1000, m=100$.

case $m=100,000$, so a weak sorting is assumed. Since $V_{\text {total }}$ is constant during the simulation (no material is disappearing from the system), the task is to consecutively choose potential $\mathbf{z}$ particles from the whole particle population until the cumulative volume of the chosen particles reaches the constant $V_{\text {total }} / m$. When this happens, the number of potential $\mathbf{z}$ particles is registered and denoted by $k$, as above.

\section{Model Results and Discussion}

\subsection{Interpretation of the Simulation Results}

[44] Model results are presented together with field results in Figure 5. The two parameters $n$ and $m$ were fitted to the field data to obtain the best agreement. Observe that the simulation results fit to the field results very well using parameters $n=1000$ and $m=100,000$, as not only do the mean values agree but also the standard deviation of the axis ratios and the maximum particle sizes match well.

[45] Since the amount of abraded material is proportional to the $(3 / 2)$ th power of particle mass (cf., equations (10)-(11)) and particle mass decreases downstream, the size of the produced fragments also decreases downstream. The maximum and average produced particle size appearing in the simulation is shown in Figure 8a. In the upper reaches, where boulders collide with each other, the maximum size produced is fine gravel, while the average size of the fragments is that of very coarse sand. In the lower part of the river, the maximum fragment size decreases rapidly and the average fragment size changes to medium and fine-grained sand. Note that because the newly created particles are smaller than $20 \mathrm{~mm}$, they do not affect the shape and size distributions computed only for the particles larger than $20 \mathrm{~mm}$.

[46] Since fragments appear rapidly in the simulated particle population, more and more particles have to be chosen to reach the constant $V_{\text {total }} / m$ in the segregation model, thus $k$ increases along the river (Figure 8b, left axis). However, the number of fragments increases more rapidly than $k$; therefore, the ratio $k / N$ between $k$ and the total number of particles $N$, decreases abruptly and converges to a small constant value (Figure 8b, right axis). This indicates that segregation by size is almost constant along the river, except the first few kilometers where it has a more important effect. As the ratio $k / N$ decreases rapidly, segregation becomes weaker but remains effective in the simulated abrasion process. Although pebbles will almost always collide with the produced fragments, segregation increases the probability of selecting the larger fragments from the fragment distribution. This is presented in Figure 8c, where the relative frequency of collisions with particles smaller than 16,8 , and $4 \mathrm{~mm}$ are shown separately. Although the relative frequency of collisions with particles smaller than $16 \mathrm{~mm}$ increases abruptly, for the sizes 8 and $4 \mathrm{~mm}$, it increases gradually as more and more small, sandsized fragments are produced in the system (Figure 8a). The effectiveness of segregation by size on the abrasion process is considered further in a parameter study in the next subsection. As the probability of collisions with very small fragments (sand) increases, large particles will move toward flatter and thinner shapes, i.e., axis ratios $y_{1}$ and $y_{2}$ will decrease as predicted by box equations and shown in Figure 5. A more detailed picture of the shape evolution is presented in section 6.3.

[47] It is worth mentioning that size selective transport and abrasion should not be separated so strictly since it is clear that the mobility of particles controls abrasion rate and vice versa [Jerolmack et al., 2011]. Although a small particle is easier to mobilize, the time spent in movement is related to the number of collisions with other particles i.e., the abrasion rate. Since model time and thus the number of collisions is 
a)

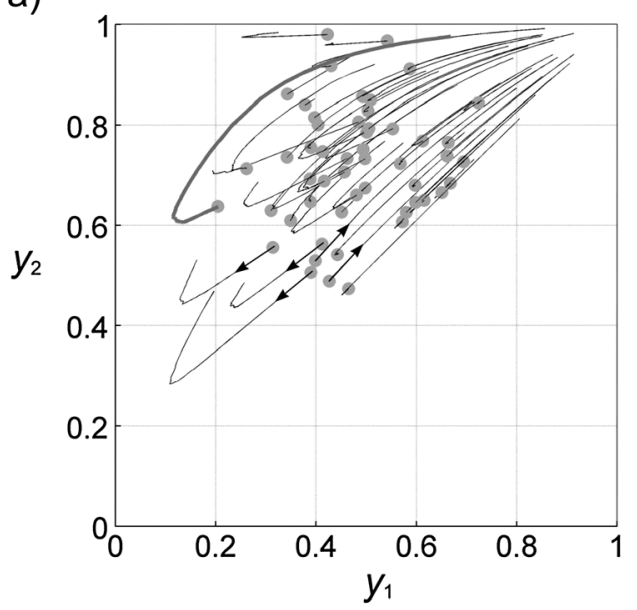

c)

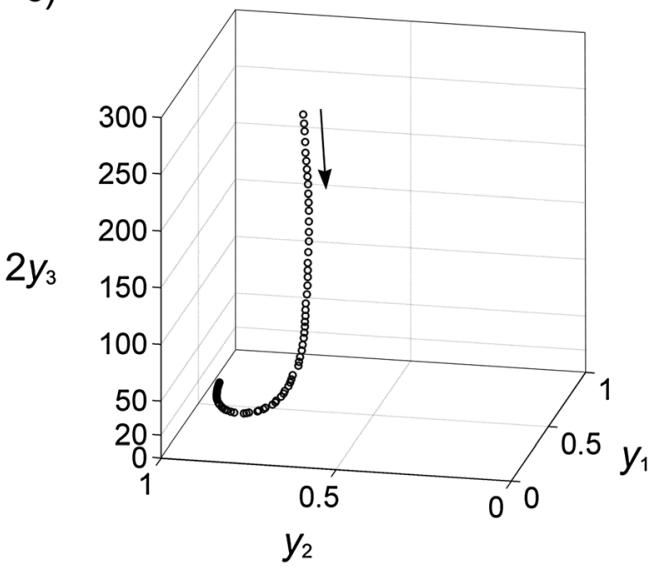

b)

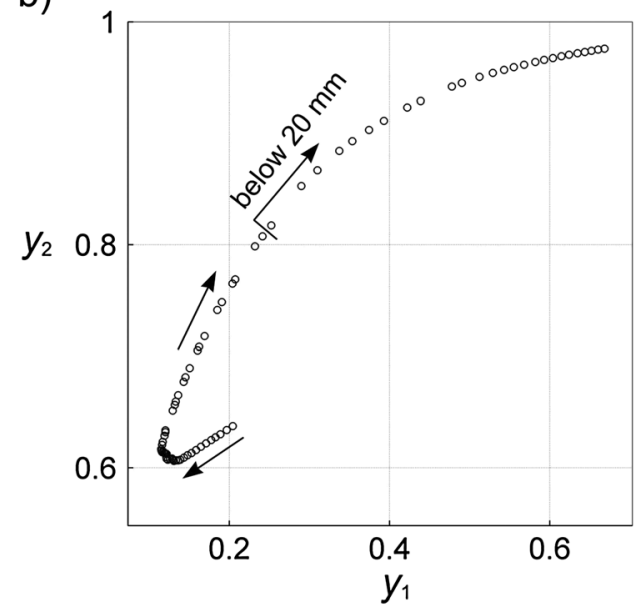

d)

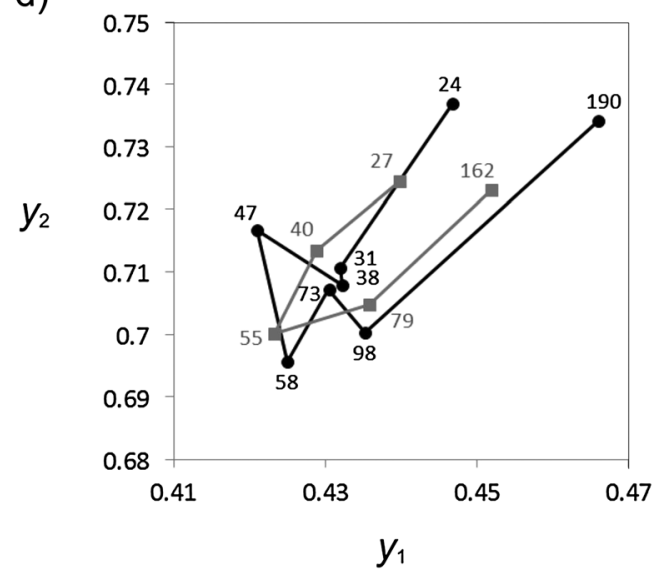

Figure 10. (a) Numerical model: trajectories of 50 randomly selected individual particles on the $y_{1}-y_{2}$ plane. Starting points are denoted by gray circles and direction is marked on a few selected curves. (b) The enlargement of one selected trajectory (the thick gray curve on Figure 10a). Shape evolution slows down around the turning point. (c) The same trajectory in the $y_{1}-y_{2}-2 y_{3}$ space. (d) Field data: data of the entire river (1626 particles) are ordered by size and averaged in this order. Numbers beside the data points refer to the average size of the particles in $\mathrm{mm}$. Black data points: data divided into eight bins, each point corresponds to 203 particles. Gray data points: data divided into five bins, each point corresponds to 325 particles.

assumed to be proportional to the distance traveled in the simulation, our model can be thought of as being ruled by the number of collisions, not by time.

\subsection{The Sensitivity of the Numerical Simulation}

[48] The sensitivity of the model results, in regard to the parameter values adopted, was also investigated and the results are presented in Figure 9. The model was run with double and half parameter values (cases A-D) and the results are shown with gray lines. The small differences between the results of cases A to D suggest that the model is structurally stable with respect to the parameters $m$ and $n$.

[49] The importance and validity of the two modeled phenomena - the production of fine particles and the effect of segregation on the abrasion - was also considered, by running the model without one or both of these phenomena present (cases $\mathrm{E}-\mathrm{G})$. The results are shown by the black lines in Figure 9. It is clear that without the production of fragments (case E), neither the shape nor the size evolution agrees with the field data. In the absence of small abraders, pebbles tend to evolve toward the sphere, as predicted by box equations. The size of large particles in the population decreases extremely slowly compared to the small particles; therefore, smaller pebbles exit from the system rather early. Accordingly, the average particle size increases in this case and the maximum particle size does not show the desirable exponential decrease. If fragment production is applied $(n=1000)$ but there is no segregation (case F), then axis ratios decrease very quickly due to the collisions with small fragments. On the other hand, if segregation is too strong (case $\mathrm{G}$ ), then particles will always collide with similar size particles, thus shapes will evolve toward the sphere.

\subsection{Prediction of the Individual Pebble Trajectories}

[50] Beyond reproducing the history of the particle population, the numerical model allows tracking of the shape and size evolution of the individual particles as well, offering a much more detailed picture of the abrasion process. 
Figure 10a presents the trajectories of 50 randomly selected particles on the $y_{1}-y_{2}$ plane, showing that a typical trajectory has a sharp U-turn on this plane. Since fragments appear rapidly in the simulated particle population, pebbles will almost always collide with small particles (Figure 8c). This can be considered as abrasion in an invariable environment, where the abraders are the fragments. In this environment, large particles start to move toward flatter and thinner shapes ( $y_{1}$ and $y_{2}$ decrease) since the Eikonal term of equations (4)-(5) dominates because of the large size difference between the abraded and the abrading particles. However, the size of the particle decreases during the abrasion process and thus the size difference between the abraded particle and the abraders (the fragments) decreases as well. This means that the roles of the second and third, curvaturedriven terms of equations (4)-(5) increase to become commensurate with the role of the Eikonal term. Therefore, shape evolution slows down on the $y_{1}-y_{2}$ plane. As the size of the abraded particle further decreases, the curvaturedriven terms start to dominate, pushing shapes toward the sphere. Therefore, when the abraded particle reaches a critical size, it turns back on the $y_{1}-y_{2}$ plane. This is presented in Figure $10 \mathrm{~b}$ on a selected trajectory. Observe that data points around the turning point are getting closer, which shows that shape evolution slows down. The same trajectory is shown in the $y_{1}-y_{2}-y_{3}$ space in Figure 10c. This phenomenon appears to be very robust: it was predicted analytically for invariable environment in Domokos and Gibbons [2012] and it has also been verified by simple laboratory experiments in their paper. They also showed that the critical size when the particle turns back depends not only on the size of the abraders but also on the axis ratios of the particles. The condition for the attractivity of the sphere, for particle $\mathbf{y}$ abraded by identical particles $\mathbf{z}$, is $\frac{\boldsymbol{y}_{3}}{z_{3}}<f\left(y_{1}, y_{2}, z_{1}, z_{2}\right)$, where $f$ is a function which depends only on the axis ratios of the abraded and abrading particles and not on their size. For spherical abraders $\left(z_{1}=z_{2}=1\right)$, the lower bound for $f$ is given by $f>3$. Thus, in the case of spherical abraders, if particle size $y_{3}$ decreases below the size $3 z_{3}$, then it is a sufficient condition for the convergence to the sphere, regardless of the axis ratios $y_{1}$ and $y_{2}$. In our case, the average size at the turning point is around $60 \mathrm{~mm}$ in the simulation (with a large standard deviation due to the differences in the axis ratios $y_{1}$ and $y_{2}$ ). Those pebbles which start with a size below the critical size do not exhibit the U-turn since their shape starts to evolve directly toward the sphere (Figure 10a). As mentioned above, the velocity along the trajectories is not constant during the abrasion process on the $y_{1}-y_{2}$ plane: around the turning point, shape evolution slows down and rapid evolution toward the sphere usually starts only below the $20 \mathrm{~mm}$ size (Figure 10b). In addition, the U-turns occur at different times for the different particles since the original particle size distribution is quite wide. This explains why the averages presented in Figure 5 do not exhibit this U-turn.

[51] However, it can be demonstrated that the field data exhibit similar behavior. To illustrate this, we treated the 12 collected samples as one particle population, i.e., we considered all of the particle data as a single sample from the entire river, as a whole. Then, we ordered the particles by size and divided them into bins in this order, so that the same number of particles fell into each bin. The mean axis ratio and size in each bin were then computed. Results are shown in the $y_{1}-y_{2}$ plane in Figure 10d where numbers beside the data points refer to the mean size in $\mathrm{mm}$. Gray/black points show the result when particles are divided into five/eight bins, respectively. The corresponding U-turn on this diagram (with an average size around $60 \mathrm{~mm}$ at the turning point) suggests that our simulation is realistic, i.e., particle size influences the abrasion process in the real river as well.

\section{Conclusions}

[52] In this paper we reviewed a new analytical abrasion model called box equations which treats the shape and size evolution of a particle population as the cumulative effect of individual collisions between two particles. The main advance of box equations is its ability to realistically model the shape evolution of pebbles together with size evolution during abrasion.

[53] We conducted a field study on the downstream evolution of basalt particle shape and size along the Williams River in the Hunter Valley, Australia. Shape changes in the downstream reaches, in particular pebbles getting flatter and thinner, suggest the importance of abrasion by suspended sediment, since according to the model predictions, this phenomenon is due to collisional abrasion by sand-sized particles. This hypothesis is strengthened qualitatively by the occurrence of aquafacts (i.e., abraded pebbles with sharp edges) at the last three sites.

[54] Based on the hypothesis that abrasion plays the dominant role in causing downstream fining in the Williams River, we presented a numerical simulation to reconstruct the abrasion process in the river. The numerical model relies on box equations, supplemented by simple geometric assumptions on fragmentation. We introduced two scalar parameters based on physical considerations: the first parameter is the number of fragments produced in each collision, while the second parameter represents the intensity of segregation by size. By fitting these two parameters, we reproduced the evolution of both the shape and size distributions along the river.

[55] Beyond reproducing field results, the model is able to follow the size and shape evolution of individual particles as well, which offers a detailed picture of the abrasion process. In particular, we demonstrated how particle size controls shape evolution in the river: large particles start to move away from the sphere; however, they turn back at a critical size and finally they converge to the sphere. While this phenomenon was predicted analytically based on box equations [Domokos and Gibbons, 2012], this is its first confirmation based on field measurements.

[56] Box equations are easy to implement and flexible. Beyond collisional abrasion, which was modeled here, the equations can handle frictional abrasion as well. Besides this, many other phenomena could be incorporated into the system, e.g., size selective transport, physical weathering or the effect of additional material from tributaries. Thus, we hope that our abrasion model, in combination with existing transport models, can provide a framework for future shape and size evolution studies in various sedimentary environments.

[57] Acknowledgments. The support of OTKA grant 104601 is gratefully acknowledged. Travel and accommodation expenses in Australia (TSZ) were supported by grant TAMOP-4.2.2.B-10/1-2010-0009, living expenses by OTKA. We are indebted to Douglas J. Jerolmack and Gary W. Gibbons for their valuable remarks and suggestions and to John Gibson for his thorough notes and his kind help in the fieldwork. We highly appreciate the detailed and 
constructive comments of Alexander Densmore, Dimitri Lague, Mikaël Attal, and two other anonymous referees. Their suggestions greatly helped us to improve the first version of the manuscript.

\section{References}

Arnold, V. I. (1986), Catastrophe Theory, $2^{\text {nd }}$ ed., Springer-Verlag, Berlin. Attal, M., and J. Lavé (2006), Changes of bedload characteristics along the Marsyandi River (central Nepal): Implications for understanding hillslope sediment supply, sediment load evolution along fluvial networks, and denudation in active orogenic belts, Spec. Pap. Geol. Soc. Am., 398, 143-171, doi:10.1130/2006.2398(09).

Attal, M., and J. Lavé (2009), Pebble abrasion during fluvial transport: Experimental results and implications for the evolution of the sediment load along rivers, J. Geophys. Res., 114, F04023, doi:10.1029/ 2009JF001328.

Bloore, F. J. (1977), The shape of pebbles, Math. Geol., 9(2), 113-122, doi:10.1007/BF02312507.

Blott, S. J., and K. Pye (2001), GRADISTAT: A grain size distribution and statistics package for the analysis of unconsolidated sediments, Earth Surf. Processes Landforms, 26(11), 1237-1248, doi:10.1002/esp.261.

Blott, S. J., and K. Pye (2008), Particle shape: A review and new methods of characterization and classification, Sedimentology, 55(1), 31-63, doi:10.1111/j.1365-3091.2007.00892.x.

Bradley, W. C. (1970), Effect of weathering on abrasion of granitic gravel, Colorado River (Texas), Geol. Soc. Am. Bull., 81(1), 61-80.

Bradley, W. C., R. K. Fahnestock, and E. T. Rowekamp (1972), Coarse sediment transport by flood flows on Knik River, Alaska, Geol. Soc. Am. Bull., 83(5), 1261-1284.

Brewer, P. A., G. J. L. Leeks, and J. Lewin (1992), Direct measurement of inchannel abrasion processes, in Erosion and Sediment Transport Monitoring Programmes in River Basins, edited by J. Bogen et al., pp. 21-30, IAHS Publication, Wallingford.

Carlston, C. W. (1969), Downstream variations in the hydraulic geometry of streams; special emphasis on mean velocity, Am. Jour. Sci., 267(4), 499-509.

Chatanantavet, P., E. Lajeunesse, G. Parker, L. Malverti, and P. Meunier (2010), Physically based model of downstream fining in bedrock streams with lateral input. Water Resour. Res., 46, W02518, doi:10.1029/ 2008WR007208.

Cui, Y., C. Paola, and G. Parker (1996), Numerical simulation of aggradation and downstream fining, J. Hydraul. Res., 34(2), 185-204, doi:10.1080/ 00221689609498496.

Dawson, M. (1988), Sediment size variation in a braided reach of the Sunwapta River, Alberta, Canada, Earth Surf. Processes Landforms, 13(7), 599-618, doi:10.1002/esp.3290130705.

Domokos, G., and G. W. Gibbons (2012), The evolution of pebble size and shape in space and time, Proc. R. Soc. A, 468(2146), 3059-3079, doi:10.1098/rspa.2011.0562.

Domokos, G., and G. W. Gibbons (2013), Geometrical and physical models of abrasion, http://arxiv.org/abs/1307.5633.

Domokos, G., A. Á. Sipos, G. M. Szabó, and P. L. Várkonyi (2009), Formation of sharp edges and planar areas of asteroids by polyhedral abrasion, Astrophys. J., 699, L13, doi:10.1088/0004-637X/699/1/L13.

Erskine, W. D. (2001), Geomorphic evaluation of past river rehabilitation works on the Williams River, New South Wales, Ecol. Manage. Restor., 2(2), 116-128, doi:10.1046/j.1442-8903.2001.00075.x.

Ferguson, R. I. (2003), Emergence of abrupt gravel to sand transitions along rivers through sorting processes, Geology, 31(2), 159-162.

Ferguson, R. I., T. Hoey, S. Wathen, and A. Werritty (1996), Field evidence for rapid downstream fining of river gravels through selective transport, Geology, 24(2), 179-182.

Firey, W. J. (1974), The shape of worn stones, Mathematika, 21(1), 1-11.

Fityus, S., A. Giacomini, and O. Buzzi (2013), The significance of geology for the morphology of potentially unstable rocks, Eng. Geol., 162, doi:10.1016/j.enggeo.2013.05.007.

Frankel, J. J. (1955), Water-faceted pebbles from Isipingo Beach, Natal, South Africa, J. Geol., 63(2), 191.

Frings, R. M. (2011), Sedimentary characteristics of the gravel-sand transition in the River Rhine, J. Sediment. Res., 81(1), 52-63, doi:10.2110/ jsr.2011.2

Greeley, R., N. T. Bridges, R. O. Kuzmin, and J. E. Laity (2002), Terrestrial analogs to wind-related features at the Viking and Pathfinder landing sites on Mars, J. Geophys. Res., 107(E1), 5005, doi:10.1029/2000JE001481.

Griffith, A. A. (1921), The phenomena of rupture and flow in solids, Philos. T. Roy. Soc. A, 221, 163-198.

Hoey, T., and R. I. Ferguson (1994), Numerical simulation of downstream fining by selective transport in gravel bed rivers: Model development and illustration, Water Resour. Res., 30(7), 2251, doi:10.1029/ 94WR00556.
Huisken, G. (1984), Flow by mean curvature of convex sources into spheres, J. Differ. Geom., 20(1), 237-266.

Jerolmack, D. J., and T. A. Brzinski (2010), Equivalence of abrupt grain-size transitions in alluvial rivers and eolian sand seas: A hypothesis, Geology, 38(8), 719-722, doi:10.1130/G30922.1.

Jerolmack D. J., M. D. Reitz, and R. L. Martin (2011), Sorting out abrasion in a gypsum dune field, J. Geophys. Res., 116, F02003, doi:10.1029/ 2010JF001821.

Kendall, K. (1978), The impossibility of comminuting small particles by compression, Nature, 272(5655), 710-711.

Knight, J. (2008), The environmental significance of ventifacts: A critical review, Earth Sci. Rev., 86, 89-105, doi:10.1016/j.earscirev.2007.08.003.

Kodama, Y. (1994), Downstream changes in the lithology and grain size of fluvial gravels, the Watarase River, Japan: Evidence of the role of abrasion in downstream fining, J. Sediment. Res., Sect. A, 64, 68-75.

Kuenen, P. H. (1947), Water-faceted boulders, Am. J. Sci., 245(12), 779-783, doi:10.2475/ajs.245.12.779.

Lajeunesse, E., L. Malverti, and F. Charru (2010), Bed load transport in turbulent flow at the grain scale: Experiments and modeling, J. Geophys. Res., 115, F04001, doi:10.1029/2009JF001628.

Lamb, M. P., W. E. Dietrich, and L. S. Sklar (2008), A model for fluvial bedrock incision by impacting suspended and bed load sediment, J. Geophys. Res., 113, F03025, doi:10.1029/2007JF000915.

Le Bouteiller, C., F. Naaim-Bouvet, N. Mathys, and J. Lavé (2011), A new framework for modeling sediment fining during transport with fragmentation and abrasion, J. Geophys. Res., 116, F03002, doi:10.1029/ 2010JF001926.

Leopold, L. B. (1953), Downstream change of velocity in rivers, Am. Jour. Sci., 251(8), 606-624.

Lewin, J., and P. A. Brewer (2002), Laboratory simulation of clast abrasion, Earth Surf. Processes Landforms, 27(2), 145-164, doi:10.1002/ esp.306.

Mikos, M. (1994), The downstream fining of gravel-bed sediments in the Alpine Rhine River, in Dynamics and Geomorphology of Mountain Rivers, edited by P. Ergenzinger and K. H. Schmidt, pp. 93-108, Springer-Verlag, Berlin.

Morris, P., and D. Williams (1999), A worldwide correlation for exponential bed particle size variation in subaerial aqueous flows, Earth Surf. Processes Landforms, 24(9), 835-847.

Paola, C., and R. Seal (1995), Grain size patchiness as a cause of selective deposition and downstream fining, Water Resour. Res., 31(5), 1395-1407, doi:10.1029/94WR02975.

Parker, G. (1991a), Selective sorting and abrasion of river gravel. I: Theory, J. Hydraul. Eng., 117(2), 131-147.

Parker, G. (1991b), Selective sorting and abrasion of river gravel, II: Application, J. Hydraul. Eng., 117(2), 150-171.

Schmeeckle, M. W., J. M. Nelson, J. Pitlick, and J. P. Bennett (2001), Interparticle collision of natural sediment grains in water, Water Resour. Res., 37, 2377-2391, doi:10.1029/2001WR000531.

Schumm, S. A., and M. A. Stevens (1973), Abrasion in place: A mechanism for rounding and size reduction of coarse sediments in rivers, Geology, 1, 37-40.

Seal, R., and C. Paola (1995), Observations of downstream fining on the North Fork Toutle River near Mount St. Helens, Washington, Water Resour. Res., 31(5), 1409-1419, doi:10.1029/94WR02976.

Sneed, E. D., and R. L. Folk (1958), Pebbles in the Lower Colorado River, Texas: A study in particle morphogenesis, J. Geol., 66(2), 114-150, doi:10.1086/626490.

Sternberg, H. (1875), Untersuchungen uber Langen-und Querprofil geschiebefuhrender Flusse, Z. Bauwes., 25, 486-506.

Surian, N. (2002), Downstream variation in grain size along an Alpine river: Analysis of controls and processes, Geomorphology, 43, 137-149, doi:10.1016/S0169-555X(01)00127-1.

Sutherland, F. L., and C. M. Fanning (2001), Gem-bearing basaltic volcanism, Barrington, New South Wales: Cenozoic evolution, based on basalt $\mathrm{K}-\mathrm{Ar}$ ages and zircon fission track and $\mathrm{U}-\mathrm{Pb}$ isotope dating, Aust. J. Earth Sci., 48(2), 221-237, doi:10.1046/j.1440-0952.2001.00851.x.

Taljaard, M. S. (1939), Note on the occurrence of faceted pebbles as products of stream-flow, Trans. Geol. Soc. South Africa, XLII, pp. 19-21.

Ueki, T. (1999), Downstream variation in particle size, form, roundness, and lithology: A case study of the Doki River, southwest Japan, Geographical Reports of Tokyo Metropolitan University, 34, 1-24.

Várkonyi, P. L., and G. Domokos (2011), A general model for collision-based abrasion, IMA J. Appl. Math., 76(1), 47-56, doi:10.1093/imamat/hxq066.

Várkonyi, P. L., and J. E. Laity (2012), Formation of surface features on ventifacts: Modeling the role of sand grains rebounding within cavities, Geomorphology, 139-140, 220-229, doi:10.1016/j.geomorph.2011.10.021.

Wolman, M. G. (1954), A method of sampling coarse river-bed material, Trans. Am. Geophys. Union, 35(6), 951-956.

Zingg, T. (1935), Beitrag zur Schotteranalyse, Schweiz. Mineral. Petrogr. Mitt., 15, 39-140. 